\title{
Pentachlorophenol and spent engine oil degradation by Mucor ramosissimus.
}

International Biodeterioration \& Biodegradation 63 (2009) 123-129

http://dx.doi.org/10.1016/j.ibiod.2008.08.001

Rafał Szewczyk \& Jerzy Długoński

Department of Biotechnology and Industrial Microbiology, Institute of Microbiology, Biotechnology and Immunology, Faculty of Biology and Environmental Protection, University of Łódź, Banacha 12/16, 90-237 Łódź, Poland, tel. 484263544 60, Fax. 484266558

Rafał Szewczyk - tel. 4842 635-44-60; fax. 4842 665-58-18, e-mail: rszewcz@biol.uni.lodz.pl

Jerzy Długoński - tel. 4842 635-44-65; fax. +4842 665-58-18, e-mail: jdlugo@biol.uni.lodz.pl

\section{Abstract}

Pentachlorophenol (PCP) has been widely used for many years and belongs to the most toxic pollutants. Spent engine oils enter environment every day in many ways. Both of them cause great environmental concern. In the present work we focused on identifying metabolites of PCP biodegradation formed in cultures of Mucor ramosissimus IM 6203 and optimization of medium composition to enhance PCP removal in the presence of engine oil acting as a carbon source.

Pentachlorophenol (PCP) to tetrachlorohydroquinone (TCHQ) transformation was the most interesting transformation conducted by the tested strain. TCHQ was further transformed to 2,3,5,6-TCP and 2,3,4,6-TCP. Strain IM 6203 is also capable of PCP transformation to corresponding anisoles - pentachloromethoxybenzene (PCMB) and pentachloroethoxybenzene (PCEB). Characterization of enzymatic background involved in PCP to TCHQ transformation showed that TCHQ formation is catalyzed by inductive and cytochrome P-450 dependent enzymatic system Experiments conducted on mineral medium allowed defining the optimal quantitative and qualitative medium make-up for PCP to TCHQ transformation. Biodegradation of PCP on optimized synthetic medium X was more efficient than on rich Sabouraud medium. The tested strain is capable of growth in the presence of spent engine oil therefore we checked the ability of PCP transformation on optimized synthetic medium containing oil as a carbon source. Collected results showed that PCP removal and TCHQ formation was the most efficient on the oil-containing medium (OX medium). PCP removal and TCHQ forming after $240 \mathrm{~h}$ of culturing reached $1.19 \mathrm{mg} / \mathrm{l}$ and $0.89 \mathrm{mg} / \mathrm{l}$, respectively. Additionally, $55.5 \%$ of oil introduced to medium was removed during 10 days of the experiment.

PCP biodegradation mechanisms used by Mucor species are still not sufficiently explained. The presented results point to the tested strain as an interesting model for the research on fungal PCP biodegradation in the areas highly contaminated with engine oil and for its future application in PCP and oils removal.

Keywords: pentachlorophenol, spent oil, biodegradation, fungi, mucor, cytochrome p-450

\section{Introduction}

Pentachlorophenol (PCP) is a xenobiotic causing great environmental concern. It has been commonly applied for many years as a bactericide, fungicide, defoliant, herbicide, wood preservative and detergent supplement in soaps (Abramovitch and Capracotta 2003; Kot-Wasik et al. 2004; Machera et al. 1997; Nascimento et al. 2004). This biocide is slightly soluble in water, up to $12-14 \mathrm{mg} / \mathrm{l}$, and very resistant to biotic and abiotic attack, which leads to a constant increase in its concentration in soil, water sediments and living organisms.

Fuel oils are commonly used everyday in many areas. Despite large improvement in handling, transportation and containment they still enter water and soil environments. The most serious damage to natural ecosystems was reported after accidental releases (Chaineau et al. 2005). Oils are a mixture of simple and complex, aliphatic or aromatic hydrocarbons which are toxic to humans, plants and animals (Van Hamme and Ward 2001). They can be removed by physical methods such as evaporation, photooxidation, washed from the atmosphere and soil with rain or various chemical reactions (Arzayus et al. 2001; Hurley et al. 2001; Sharma et al. 2002; Garrett et al. 2003). In case of removal from the environment microbial biodegradation is sometimes the only way, especially for the nonvolatile components (Venosa and Zhu 2003). Microorganisms capable of petroleum and oils removal originate from areas of recent or chronic oil contamination. In such environments, they can constitute up to $100 \%$ of the viable microorganisms (Atlas 1981; Venosa and Zhu 2003). Oil biodegradation in nature involves a succession of species including consortia of microbes (Venosa and Zhu 2003). In spite of physical and biological processes, oil components may persist for a long time in soil and water sediments (Chaineau et al. 2000).

The knowledge on pathways of PCP biodegradation by fungal species is still limited. PCP is a highly hydrophobic compound and it tends to accumulate in soil or water sediments with many other hydrophobic xenobiotics. There have been reports on many areas contaminated with oils, diesel fuels or PAHs, PCP and other chlorophenols (Virendra and Pendey 2004, Jiayin et al 2007, Götz et al 2007). In our preceding work (Szewczyk et al. 2003) we isolated from spent cutting fluid a fungal strain Mucor ramosissimus IM 6203 which demonstrated the ability of PCP $(10 \mathrm{mg} / \mathrm{ll})$ to $\mathrm{TCHQ}$ transformation on oil containing medium. In the present study we focused on identifying PCP biodegradation byproducts, enzymatic background and the role of medium ingredients in biodegradation of PCP in the presence of spent engine oil in M. ramosissimus IM 6203 cultures. Deeper insight into these aspects of the tested strain abilities presented in this work may act as a starting point for its future application in PCP and engine oil removal.

\section{Materials and methods}

\subsection{Chemicals}

Xenobiotic substrates: pentachlorobenzene (PCB) (Riedel-de Haen, 99.8\%), pentachlorophenol (PCP) (Sigma, 99.8\%), Tetrachlorohydroquinone (TCHQ) (Chem Service, 98.7\%), 2,3,5,6tetrachlorophenol (2,3,5,6-TCP) (Supelco, 99.8\%), 2,3,4,5tetrachlorophenol (2,3,4,5-TCP) (Supelco, 99.8\%), 2,3,4,6tetrachlorophenol (2,3,4,6-TCP) (Riedel-de Haen, 99.9\%), hexachlorobenzene - internal standard (Institute of Organic and Industrial Chemistry, Warsaw). Cytochrome P-450 inhibitors: 1 aminobenzotriazole (Sigma), metyrapone - 2-metylo-1,2-di-pirydylo1-propanon (98\%, Aldrich). Other chemicals: ethyl acetate, hexane, methanol, ethanol, glucose were obtained from J. T. Baker or $\mathrm{POCH}$. All the chemicals were high purity grade reagents. Spent engine oil was obtained from a car service unit in Łódź.

\subsection{Stock solutions}

Stock solutions of chlorophenols were made up to $5 \mathrm{mg} / \mathrm{ml}$ with $95 \%$ $\mathrm{v} / \mathrm{v}$ ethanol and diluted to suitable concentrations for each experiment.

\subsection{Microorganisms}

Mucor ramosissimus IM 6203 strain isolated from spent cutting fluid, was identified by standard diagnostic methods by the Department of Plant Taxonomy and Geography, University of Warsaw (Poland).

\subsection{Dry weight determination}

Filters were dried out at $105^{\circ} \mathrm{C}$ till constant weight. Samples were filtered and washed three times with distilled water. Filtered mycelium were than dried out at $105^{\circ} \mathrm{C}$ till reaching constant weight.

\subsection{Preculturing of fungal strains and cultivation on Sabouraud medium}

Fungal cultures (10 day-old) on ZT (Wilmańska et al. 1992) agar slants were used to prepare inoculum for $20 \mathrm{ml}$ Sabouraud liquid medium (Difco). Preculturing (24h) and main incubation were carried out at $28{ }^{\circ} \mathrm{C}$ on rotary shaker at $180 \mathrm{rpm}$ in $100 \mathrm{ml}$ Erlenmeyer flasks on Sabouraud liquid medium. 


\subsection{Mineral liquid media}

Synthetic medium X composition: $\left(\mathrm{NH}_{4}\right)_{2} \mathrm{HPO}_{4}-2,6 \mathrm{~g}, \mathrm{MgSO}_{4} \cdot 7 \mathrm{H}_{2} \mathrm{O}$ - 0,2g, $\mathrm{MnSO}_{4}-0,05 \mathrm{~g}, \mathrm{FeSO}_{4} \cdot 7 \mathrm{H}_{2} \mathrm{O}-0,01 \mathrm{~g}, \mathrm{CaCl}_{2} \cdot 2 \mathrm{H}_{2} \mathrm{O}-0,03 \mathrm{~g}$. glucose - $50 \mathrm{~g}$ and distilled water up to $1000 \mathrm{ml}$. Medium OX composition: $\left(\mathrm{NH}_{4}\right)_{2} \mathrm{HPO}_{4}-2,6 \mathrm{~g}, \mathrm{MgSO}_{4} \cdot 7 \mathrm{H}_{2} \mathrm{O}-0,2 \mathrm{~g}, \mathrm{MnSO}_{4}-$ $0,05 \mathrm{~g}, \mathrm{FeSO}_{4} \cdot 7 \mathrm{H}_{2} \mathrm{O}-0,01 \mathrm{~g}, \mathrm{CaCl}_{2} \cdot 2 \mathrm{H}_{2} \mathrm{O}-0,03 \mathrm{~g}$. spent engine oil $-5 \%$ and distilled water up to $1000 \mathrm{ml}$. The $\mathrm{pH}$ of all liquid media was 6.8 .

\subsection{Culturing parameters}

Inoculum ( $24 \mathrm{~h}$ old culture obtained as described above) was introduced into tested medium with PCP (10 mg/l) (inoculum: medium ratio=1:9). The flasks were incubated for 7 or 10 days, at 28 ${ }^{\circ} \mathrm{C}$ on rotary shaker (180 rpm). After incubation periods (depending on the experiment) samples for dry weight, chlorophenols content or oil content were collected. Uninoculated xenobiotic-containing media served as abiotic controls and inoculated ones without xenobiotic addition served as growth controls.

\subsection{Extraction of xenobiotics}

The samples were filtered. The mycelium was suspended in water and disintegrated using sonicator (MISONIX, England). Then fungal homogenates and culture filtrates were three times extracted separately with ethyl acetate. The extracts were dried over anhydrous sodium sulphate and the solvents were evaporated under reduced pressure at $40^{\circ} \mathrm{C}$.

\subsection{Chromatography methods}

Chromatography methods were based on modified ones previously described in our preceding work (Szewczyk et al. 2003). Gas chromatographic analyses of ethyl acetate extracts were performed on a Hewlett-Packard HP 6890 series gas chromatograph equipped with mass selective detector HP 5973, using a HP-5MS capillary column. The injection volume was $2 \mu \mathrm{l}$. The inlet was set to split mode with split ratio 10:1 (split flow $10 \mathrm{ml} / \mathrm{min}$ ) and temperature maintained at $250{ }^{\circ} \mathrm{C}$. Helium was used as a carrier gas. Temperature parameters of the column were as follows: $80^{\circ} \mathrm{C}$ maintained for 1 minute, $20^{\circ} \mathrm{C} / \mathrm{min}$ to $220^{\circ} \mathrm{C}, 5^{\circ} \mathrm{C} / \mathrm{min}$ to $230^{\circ} \mathrm{C}$, $20^{\circ} \mathrm{C} / \mathrm{min}$ to $290^{\circ} \mathrm{C}$ maintained for 3 minutes. For the determination of $\mathrm{PCP} / \mathrm{TCHQ}$ and oil content on $\mathrm{OX}$ medium temperature parameters of the column were as follows: $80^{\circ} \mathrm{C}$ maintained for 1 minute, $20^{\circ} \mathrm{C} / \mathrm{min}$ to $220^{\circ} \mathrm{C}, 5^{\circ} \mathrm{C} / \mathrm{min}$ to $300^{\circ} \mathrm{C}$ maintained for 20 minutes. Column carrier gas constant flow was $1 \mathrm{ml} / \mathrm{min}$. The column to MS aux channel temperature was maintained at $250{ }^{\circ} \mathrm{C}$ for 10 minutes and then increased to $290^{\circ} \mathrm{C}$ with ratio $15^{\circ} \mathrm{C} / \mathrm{min}$. Mass selective detector parameters were as follows: ms source 230 ${ }^{\circ} \mathrm{C}$, ms quad $150{ }^{\circ} \mathrm{C}$, scan mode with mass range set from 45.0 to 350.0. All methoxy-products were identified only on the basis of the comparison of the retention time and mass spectrum of the examined sample to the retention time and mass spectrum in database (NIST MS Chemstation Library) attached to GC-MS, which allowed positing that the probability of its presence in the extracts amounts to $90-95 \%$. Other compounds were assayed on the basis of the comparison of the retention time and mass spectrum of the examined sample to the retention time and mass spectrum of the reference standard and the retention time and mass spectrum in database (NIST MS Chemstation Library) which allowed positing that the probability of their presence in the extracts amounted to $99 \%$. Oil content was measured on the basis of peaks area summary between 3 and 28 minute of chromatographic analysis.

\subsection{Characterization of enzymes involved in $P C P$ to $T C H Q$ transformation}

1. Cytochrome P-450 involvement: To Erlenmeyer flasks containing $18 \mathrm{ml}$ of Sabouraud medium and $2 \mathrm{ml}$ of $24 \mathrm{~h}$ of inoculum, PCP (10 $\mathrm{mg} / \mathrm{l})$, 1-aminobenzotriazole $(1 \mathrm{mM})$ or metyrapone $(2 \mathrm{mM})$ were added in $0 \mathrm{~h}$ or $24 \mathrm{~h}$ of culturing. Cultures were incubated for 5 days from the day of substrate addition (Lisowska and Długoński 2003). Samples for xenobiotic and its derivatives content and dry weight were collected (as described above).

2. PCP transformation in starvation conditions: To Erlenmeyer flasks containing $18 \mathrm{ml}$ of Sabouraud medium and $2 \mathrm{ml}$ of $24 \mathrm{~h}$ old inoculum. Cultures were incubated on rotary shaker (180 rpm) at $28^{\circ} \mathrm{C}$ for $24 \mathrm{~h}$. After $24 \mathrm{~h}$ of incubation $0.1 \mathrm{mg} / \mathrm{l}$ of PCP was added to flasks and cultured for another $24 \mathrm{~h}$. After $24 \mathrm{~h}$ of incubation whole culture $(20 \mathrm{ml})$ was filtered in aseptic conditions, washed three times with aseptic distilled water. Obtained mycelium was transferred to Erlenmeyer flasks with $20 \mathrm{ml}$ aseptic distilled water and PCP (10 $\mathrm{mg} / \mathrm{l})$. Obtained cultures were incubated for 7 days and samples for $\mathrm{PCP} / \mathrm{TCHQ}$ and dry weight determination were collected as described above. Between 0 and $48 \mathrm{~h}$ of culturing the presented result is an average result from three independent experiments.

\section{Results}

\subsection{Pentachlorophenol transformation}

Examined fungal strain M. ramosissimus IM 6203 is capable of pentachlorophenol transformation to tetrachlorohydroquinone (TCHQ) (Szewczyk et al. 2003). In the preliminary experiments we focused on looking for other metabolites of PCP transformation in the cultures conducted on rich Sabouraud medium which is the most preferable one for the growth of tested fungal strain. The qualitative tests were made according to revised GC-MS method applied in the previous manuscript (Szewczyk et al. 2003). Table 1 shows chlorophenols transformation derivatives and their relative concentrations determined by gas chromatography and mass spectra analysis in 7-day old cultures.

As it is shown in Table 1, after 7-days of incubation TCHQ is the metabolite that occurs in the highest relative to PCP concentration reaching $12 \%$. The other ones although present, appear in relatively low concentrations - 2,3,5,6-TCP - 1\%, 2,3,4,6-TCP, PCMB and PCEB $-0,1 \%$.

Table 1. Summary of mass spectra of chlorophenols transformation derivatives and its relative concentrations in cultures Mucor ramosissimus IM 6203. Presented results are means from four separate experiments.

\begin{tabular}{|c|c|c|c|c|}
\hline Substrate & $\begin{array}{l}\text { Transformation } \\
\text { product }\end{array}$ & $\begin{array}{l}\text { Transformation } \\
\text { product } \\
\text { retention time } \\
\text { (min.) }\end{array}$ & $\begin{array}{c}\text { Transformation } \\
\text { product main ions } \\
\text { mass spectrum } \mathrm{m} / \mathrm{z} \\
\text { (relative intensity) }\end{array}$ & $\begin{array}{l}\text { Relative product to } \\
\text { substrate } \\
\text { concentration (\%) }\end{array}$ \\
\hline PCB & PCP & 8.80 & $\begin{array}{l}266(100), 165(28), \\
202(18), 230(14,5)\end{array}$ & $90 \%$ \\
\hline \multirow{2}{*}{ PCP } & $\mathrm{TCHQ}$ & 8.87 & $\begin{array}{l}248(100), 86(27), 147 \\
(26), 212(10,1)\end{array}$ & $12 \%$ \\
\hline & 2,3,5,6-TCP & 7.52 & $\begin{array}{l}232(100), 131 \text { (26), } \\
166(18), 194(13)\end{array}$ & $1 \%$ \\
\hline PCP & 2,3,4,6-TCP & 7.55 & $\begin{array}{l}232(100), 131(28,2), \\
166(22), 196(14)\end{array}$ & $0.1 \%$ \\
\hline PCP & PCMB & 8.27 & $\begin{array}{l}280(100), 265(75), \\
237(72), 167(38)\end{array}$ & $0.1 \%$ \\
\hline PCP & PCEB & 8.29 & $\begin{array}{l}266(100), 165(28), \\
237(17), 294(14)\end{array}$ & $0.1 \%$ \\
\hline $\mathrm{TCHQ}$ & $2,3,5,6-\mathrm{TCP}$ & 7.52 & as above & $40 \%$ \\
\hline 2,3,5,6-TCP & 2,3,5,6-TCMB & 7.88 & $\begin{array}{l}246(100), 203(69), \\
231(47), 131(30)\end{array}$ & $50 \%$ \\
\hline $2,3,4,5-\mathrm{TCP}$ & $X$ & - & - & - \\
\hline $2,3,4,6-\mathrm{TCP}$ & $X$ & 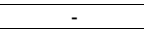 & - & - \\
\hline
\end{tabular}

$\mathrm{X}-$ no derivative found

\subsection{Enzymatic background investigation}

TCHQ was the most intensively produced metabolite of PCP in cultures of Mucor ramosissimus IM 6203, therefore we attempted to characterize the enzymatic background involved in this reaction. To determine if cytochrome $\mathrm{P}-450$ plays an important role in PCP to TCHQ transformation we cultured the tested strain with PCP and two cytochrome P-450 inhibitors: 1-aminobenzotriazole and metyrapone (Lisowska and Długoński 2003). Additionally, we conducted experiments in starvation conditions to determine inductive properties of investigated enzymes.

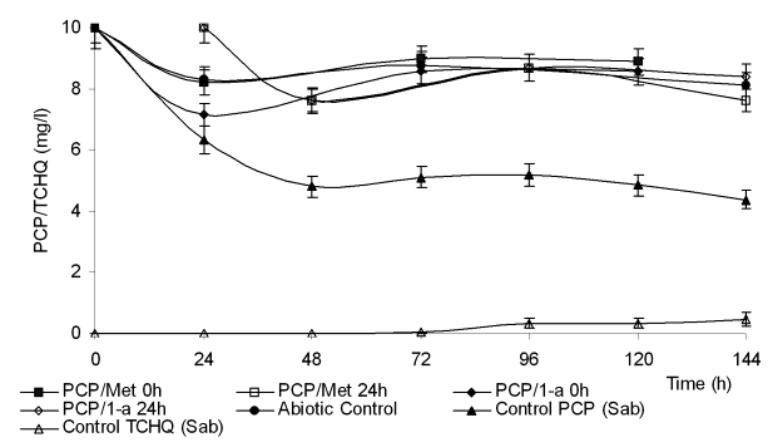

Figure 1. PCP transformation on Sabouraud medium with Metyrapone (Met) or 1aminobenzotriazloe (1-a) addition at $0 \mathrm{~h}$ or $24 \mathrm{~h}$ of culturing. Presented results are means from three replicated experiments.

Control experiments carried out on Sabouraud medium showed that, $\mathrm{TCHQ}$ formation starts from $96 \mathrm{~h}$ of culturing and reaches its 
maximum $(0.464 \mathrm{mg} / \mathrm{l})$ at the end of the experiment. PCP is rapidly absorbed by the growing mycelium during the first $48 \mathrm{~h}$ of the experiment to a level equal to $\pm 4.8 \mathrm{mg} / \mathrm{l}$. Pesticide concentration is slightly falling down from $48 \mathrm{~h}$ to $144 \mathrm{~h}$ of culturing, which corresponds with the TCHQ concentration increment mentioned above (Fig. 1). Introducing cytochrome P-450 inhibitors to the media caused in all experiments decrease or slow down of fungal growth yet presence of 1-a in cultures without PCP did not have a negative influence on the growth of the tested strain. GC-MS analysis (Fig. 1) revealed that in cultures containing inhibitors of cytochrome P-450 and PCP only an insignificant substrate loss was observed irrespective of the time of pesticide or inhibitors addition. TCHQ or any other metabolites of PCP transformation was not observed in any of the inhibitors containing cultures.

In cultures with PCP addition conducted in starvation conditions, neither growth nor significant biomass loss was observed during 7 days of incubation. Chromatography analysis of extracts showed that overall xenobiotic concentration drops down in cultures not induced and previously induced with PCP. The observed PCP loss effect may be a result of substrate to mycelium binding process intensified in starvation conditions of cultures. TCHQ presence was observed from $12 \mathrm{~h}$ to $36 \mathrm{~h}$ of culturing of PCP-induced mycelia. Maximum TCHQ concentration reaching $0.45 \mathrm{mg} / \mathrm{l}$ was determined in $18 \mathrm{~h}$ of the experiment (Fig. 2).

The obtained results show that the process of $\mathrm{PCP}$ to $\mathrm{TCHQ}$ transformation by Mucor ramosissimus IM 6203 is conducted by an inductive enzymatic system associated with cytochrome P-450.

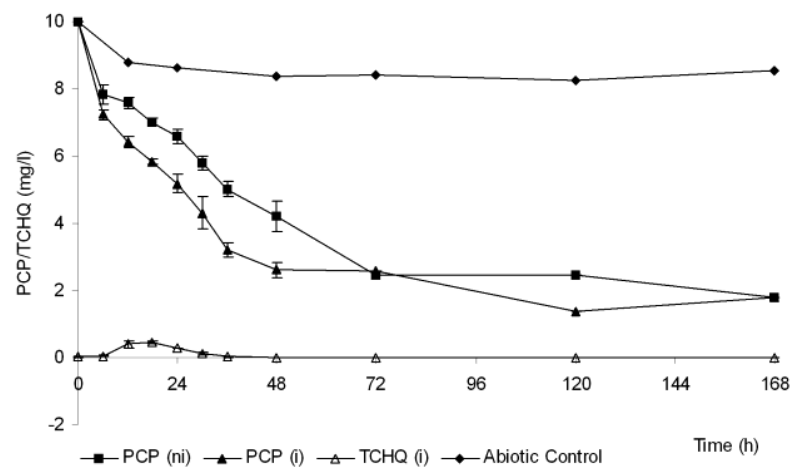

Figure 2. PCP transformation by Mucor ramosissimus IM 6203 in starvation conditions: (ni) mycelium not induced with PCP; (i) - mycelium induced with PCP. Presented results are means from three replicated experiments.

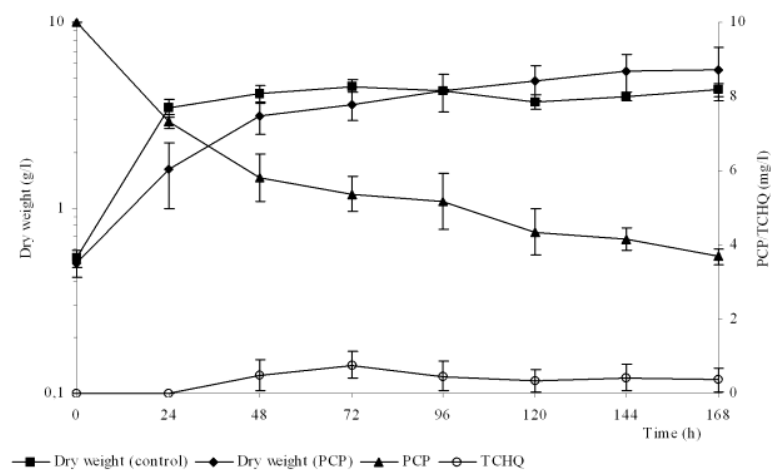

Figure 3. Dry weight, PCP and TCHQ content in cultures of Mucor ramosissimus IM 6203 on synthetic medium X. Presented results are means from three replicated experiments

\subsection{PCP biodegradation on synthetic medium $X$}

In the following step of the research we focused on PCP removal on synthetic medium $X$ with exact quantitative and qualitative make-up and composed on the basis of mineral medium developed by Lobos et al. (1992). Synthetic medium X was optimized for growth and PCP degradation by the tested strain (data not shown). Mycelium growth in cultures with xenobiotic addition was slower than in control medium, however, in the last hours of culturing in flasks containing PCP, increment of the biomass was observed (Fig. 3).

PCP to TCHQ transformation starts between 24 and 48 hour of incubation. During the time of culturing systematic decrease in PCP content is observed (from $10 \mathrm{mg} / \mathrm{l}$ in $0 \mathrm{~h}$ to $4.63 \mathrm{~g} / \mathrm{l}$ in 7 day of incubation), and maximal recovery of TCHQ was stated in $72 \mathrm{~h}$ of culturing (0.76 mg/l) (Fig. 3). Growth of strain Mucor ramosissimus IM 6203 in the presence of PCP is delayed in comparison to control system without pesticide addition and during the first $24 \mathrm{~h}$ of incubation, no products of PCP biodegradation were observed.

\subsection{PCP biodegradation on medium $O X$}

To determine how the optimal mineral medium composition would enhance PCP biodegradation in spent oil presence we checked the ability of xenobiotic biodegradation in medium OX containing $5 \%$ of spent engine oil dispersed in glucose absent synthetic medium $\mathrm{X}$. Biomass determination in this medium was relatively difficult to handle, therefore in this case we used only microscopic and macroscopic observations for characterisation of the culture growth. Mycelium growth was observed from $72 \mathrm{~h}$ of incubation and reached its relative maximum between 168 and 240 hour of culturing. The lack of growth in the first $72 \mathrm{~h}$ hours of culture was probably caused by the microorganism adaptation to medium composition.

GC-MS results showed that during the first $72 \mathrm{~h}$ of culturing PCP concentration decreased to $5.25 \mathrm{mg} / \mathrm{l}$. Xenobiotic content was further decreasing systematically till the end of the experiment in $240 \mathrm{~h}$ of incubation reaching only $1.19 \mathrm{mg} / \mathrm{l}$. TCHQ presence was observed from 72 hour of incubation $(0.28 \mathrm{mg} /)$. Maximum concentration of $\mathrm{TCHQ}-0,89 \mathrm{mg} / \mathrm{l}$ was observed in $240 \mathrm{~h}$ of the experiment. Chromatographic analysis also allowed determining the oil content decrease on the basis of peaks area summary in the following hours of culturing. As it is shown in Fig. 4, the maximum PCP and oil decrease coupled with maximum TCHQ production was observed from the 168 to $240 \mathrm{~h}$ of incubation. At the end of culturing $55.5 \%$ of the initial oil concentration was removed. Macroscopic observations showed that the most dynamic mycelium growth was observed during the last three days of the experiment.

Collected results shows that tested strain is a very interesting tool for simultaneous PCP and spent diesel oils biodegradation. PCP transformation and removal is even more effective in a heavily contaminated with engine oils environment than on the rich Sabouraud or synthetic X media.

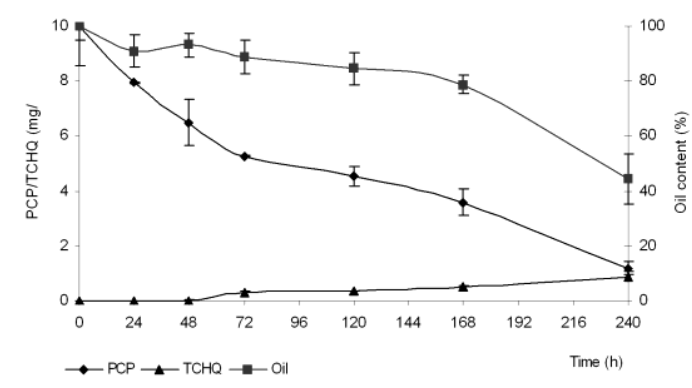

Figure 4. PCP and overworked engine oil biodegradation by Mucor ramosissimus IM 6203 , during $240 \mathrm{~h}$ of incubation on OX medium. Presented results are means from three replicated experiments.

\section{Discussion}

Aerobic biodegradation of chlorophenols may occur in many different ways. The key reaction depends on inserting hydroxyl groups to the aromatic ring of chlorophenol with simultaneous removal of chlorine atoms or reductive dechlorination (van Pee and Unversucht 2003).

According to the results, two different ways of chlorophenols transformation by M. ramosissimus IM 6203 were observed. One way involved hydroxyl group insertion to aromatic ring with simultaneous chlorine removal and the second one involved chlorophenols hydroxyl group methylation resulting in a formation of anisoles (Fig. 5). The observed transformations occurred with a different intensity as shown in Table 1. In some cases in cultures with PCP addition different derivatives were observed. Observed in all experiments $\mathrm{PCP}$ to $\mathrm{TCHQ}$ transformation was the most intensive reaction. TCHQ was transformed further by hydroxyl group removal in the 4 position to 2,3,5,6-TCP (Fig. 5). Many authors point to $\mathrm{TCHQ}$ as the main metabolite of PCP biodegradation. Copley (2000) reported that TCHQ was converted to $2,4,6-\mathrm{TCP}$. Trichlorophenol is also the next step by-product of TCHQ biodegradation in Pchanerochaete chrysosporium cultures (Reddy and Gold 1999), but in our study we only found 2,3,5,6-TCP. Degradation of PCP is a step by step process of removing consecutive chlorine atoms which leads to 2,6-DCP, which is further metabolized by ring-cleavage, and final oxidation of metabolites in the Krebs cycle (Janssen et al. 1994; Webb et al. 2001). Law et al. (2003) proposed an alternative pathway of complete PCP degradation in oxygen conditions carried out by consortia of microorganisms settled in the compost previously used in the culturing of Pleurotus pulmonarius. This process depends on successive dechlorinations with gaseous chlorine release, leading to a formation of phenol, toluenes, phtalans and finally octadecanoic acid and hexadecane. 
Mass spectrum analysis carried out in this research suggested that strain M. ramosissimus IM 6203 also produced 2,3,4,6-TCP in a trace concentration. A similar reaction was described by Shim and Kawamoto (2002), where 2,3,4,6-TCP was the main metabolite in PCP biodegradation by $P$. chrysosporium. In oxygen conditions a process of chlorophenols conjugation may also be observed, but we did not find any derivatives or compound fragments suggesting the presence of this kind of metabolites. Webb et al. (2001) said that PCP was transformed by Saccharomonospora viridis in the way similar to the one certified in mammals, which involved the conjugation of $\mathrm{TCHQ}$ formed in the culture with compounds containing -SH group, for example: glutathione or cysteine.

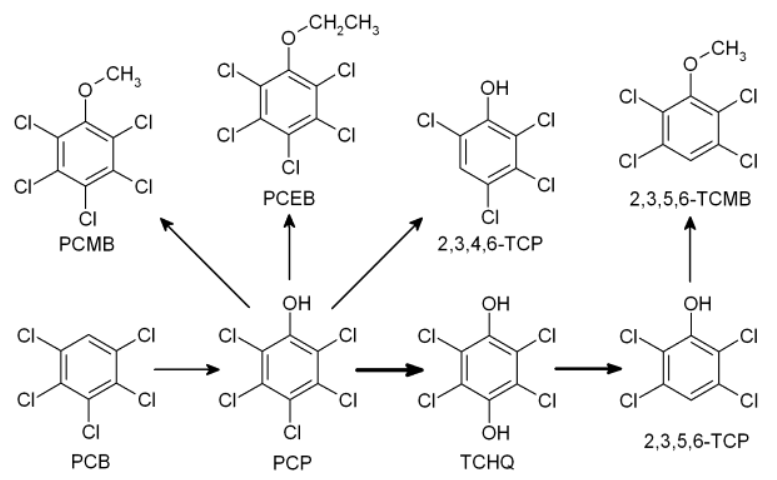

Figure 5. Reaction scheme of chlorophenols transformation by Mucor ramosissimus IM 6203 .

Bolded arrows mark reactions that took place in experiments with PCP addition.

Other transformations of polichlorophenols lead to the reduction of toxicity of the substrate without chlorine atoms removal. The most common transformation of this type in oxygen conditions is methylation or ethylation of the hydroxyl group in chlorophenols compounds. These reactions lead to the formation of chloroanisoles - compounds less reactive and toxic than their substrates, but much more recalcitrant to microbiological attack. Their hydrophobic properties also increase (Lamar and Dietrich 1990; Lamar et al. 1990; Lestan and Lamar 1996). In case of strain M. ramosissimus IM 6203 , these seem to be only side reactions that occur with a trace intensity giving two methoxyderivatives

pentachloromethoxybenzene (PCMB) and pentachloroethoxybenzene (PCEB) (Fig. 5). O-methylation reaction of many chlorophenols is conducted by Trichoderma longibrachiatum (Coque et al. 2003). Alvarez-Rodriguez et al. (2002) isolated 15 strains of fungi capable of O-methylation of 2,4,6-TCP. Two of them belong to genus Trichoderma, one to genus Mucor. A formation of anisoles was observed in white rot fungus Phanerochaete sp. cultures (Lamar et al. 1990; Lestan and Lamar 1996) and also in higher organisms (Bollag 1992).

Xenobiotics biodegradation may be catalyzed by various groups of enzymes, for example: lignolitic enzymes, dioxygenases, monoxygenases, methylotransferases or dehalogenases. An important role is played by cytochrome P-450 monoxygenases, which are present in microorganisms, plants and animals. These groups of enzymes are capable of hydroxylation, epoxydation, dealkilation and dehalogenation of heterogeneous groups of xenobiotics (Kellner et al. 1997). Chlorophenols hydroxylation in para position is quite a common reaction in the pathways of these compounds group degradation (Janssen et al. 1994). The results obtained in this work showed that the process of PCP to TCHQ transformation by Mucor ramosissimus IM 6203 is conducted by an inductive enzymatic system associated with cytochrome P-450. The relation between orto hydroxylation of 2,3,5-TCP by $R$. opacus $1 \mathrm{G}$ and cytochrome P-450 was suggested by Bondar et al. (1999). Similar process was described in higher organisms in the research on purified rats and mice liver cells was conducted by Tsai et al. (2001). P-450 monooxygenase catalyses orto and para hydroxylation of PCP. Formed hydroxychlorophenols are then transformed to quinones by cytochrome $\mathrm{P}-450$ quinone reductase. Copley (2000) described PCP degradation by S. chlorophenolica. The TCHQ was the first and crucial metabolite of PCP degradation pathway. The described enzyme is not specific and may catalyze hydroxylation reactions of different chlorophenols. Le Garrec et al. (2001) described catalitycal properties of PCP inducible chlorophenol 4-monoxygenase isolated from B. cepacia AC1100 responsible for para hydroxylation of chlorophenols.

Experiments on synthetic medium $X$ showed that mycelium growth in cultures with xenobiotic addition was suppressed in the first hours of culturing, however, at the end of experiment in flasks containing PCP, increment of the biomass was observed (Fig. 3). The obtained result may point to the possibility of a potential use of the xenobiotic or its unidentified derivative for building mycelium of $M u c o r$ ramosissimus IM 6203 because in the final hours of the experiment only PCP concentration is reduced. On the other hand, the quantity of PCP introduced to medium is not enough for explaining biomass growth. Probably the presence of xenobiotic in cultures affects mycelium physiology as well as the manner of medium components utilization and use. A systematic decrease in PCP content in the culture is observed (from $10 \mathrm{mg} / \mathrm{l}$ in $0 \mathrm{~h}$ to $4.63 \mathrm{~g} / \mathrm{l}$ in 7 day of incubation) and maximum recovery of $\mathrm{TCHQ}$ was stated in $72 \mathrm{~h}$ of experiment $(0.76 \mathrm{mg} / \mathrm{l})(\mathrm{Fig} .3)$. The obtained results suggested, that the increase in TCHQ concentration observed between 48 and $72 \mathrm{~h}$ was related to Mucor ramosissimus IM 6203 growth, cometabolic reactions and stationary phase metabolism of the tested strain. The most intensive removal of PCP by Rhizopus nigricans took place till $24 \mathrm{~h}$ of the culture, which was strictly coupled with the culture growth in the research conducted by Cortes et al. (2002). Analogical results were obtained by Webb et al. (2001) in cultures of Saccharomonospora viridis with PCP addition, where the decline of substrate content took place during the logarithmic phase of growth. Mucor ramosissimus IM 6203 growth in cultures with PCP addition is delayed in comparison to the control system. It may suggest a possibility of other factors determining PCP transformation. They might be, adaptation of microorganism to toxic substrate or competition between primary metabolism (mycelium growth) and oxidative dehalogenation of xenobiotic (transformation of PCP to $\mathrm{TCHQ}$.

PCP is a hydrophobic compound which accumulates in soil or water sediments. There have been reports on many areas contaminated with oils, diesel fuels or PAHs and PCP (Virendra and Pendey 2004; Jiayin et al. 2007; Götz et al. 2007), therefore, we checked the ability of PCP $(10 \mathrm{mg} / \mathrm{l})$ biodegradation on OX medium containing $5 \%$ of spent engine acting as a carbon source. PCP removal and TCHQ formation were the most efficient on oil containing medium and strictly coupled with mycelium growth in the last hours of experiment. PCP concentration was decreasing till the end of experiment in $240 \mathrm{~h}$ of incubation and reached only $1.19 \mathrm{mg} / \mathrm{l}$. TCHQ presence was observed from 72 hour of incubation and its maximum concentration $0.89 \mathrm{mg} / \mathrm{l}$ was observed also in 240h of the experiment. Oil content decrease determined on the basis of peaks area summary was coupled with the maximum PCP removal and TCHQ production observed from the 168 to $240 \mathrm{~h}$ of incubation. The removal of xenobiotics took place during the most dynamic mycelium growth and at the end of 10-days long culturing $55.5 \%$ of initial oil concentration was removed. It has to be stated that oil utilisation was not caused by the accumulation/absorption process which was proved by the GC-MS analysis and macroscopic observations which showed that the mycelium, although hard and packed, was white, clean and oil free. Only several studies have been done on fungi biodegradation of engine oils. Most authors are focused on bacterial or mixed cultures characterization therefore fungal biodegradation of engine oils are still an open area for research. Adedokun and Ataga (2006) characterized growth of three edible mushrooms on solid media containing crude and spent oils in various concentrations. One of the tested fungi, Pleurotus pulmonarius, was growing efficiently in the presence of $5 \%$ of crude or spent oil addition. Higher concentrations of oil strongly inhibited growth of all tested fungal strains. Experiments with Pleurotus tuber-regium on oilcontaminated soil (Adenipekun 2008) showed that after six months of incubation increase in nutrient content coupled with heavy metals concentration decrease was observed. Author suggested possible use of the tested mushroom in decontaminating environment polluted with engine oil. Microbial consortium in a salt marsh described by Wright and Weaver (2004) was able to remove $50 \%$ of oil contamination during 33 days of experiment. Bacteria isolated from volcanic island were able to grow in liquid cultures on mineral medium with $2 \%(\mathrm{w} / \mathrm{v})$ crude oil as the sole carbon source and were found to degrade long chain crude oil alkanes in a range between $46.64 \%$ and $87.68 \%$ after 10 days of incubation (Meintanis et al. 2006). Chaineau et al. (2005) in the 150-day experiment on crude oil biodegradation in soil by mixed culture reached the maximal biodegradation extent at $62 \%$ in an experiment with high input of mineral nutrients. Two bacterial strains isolated by Michaud et al. (2004) were capable of $85 \%$ hydrocarbons removal from diesel oil, but during 60-days period.

The applied medium OX seems to be most appropriate for PCP removal by the tested fungal strain among the media tested in this work considering the fact that it is based mostly on major concern waste - spent engine oil, and simple mineral ingredients acting primarily as $\mathrm{N}$ and $\mathrm{P}$ source and secondly as a trace metals source. Mineral nutrients input may enhance the oil biodegradation rates in soil from $47 \%$ to $62 \%$ (Chaineau et al. 2005). As it was reported by 
Shin et al. (2000) the presence of $\mathrm{N}$ sources is the most limiting factor of crude oil biodegradation in field conditions contrary to $\mathrm{P}$ presence. The addition of $\mathrm{NH}_{4}{ }^{+}$in the concentration of $100-670 \mathrm{mg} / \mathrm{l}$ efficiently stimulated degradation of crude oil in salt marsh soils. Oh et al. (2001) stated that $\mathrm{N}$ and $\mathrm{P}$ sources are the most limiting factors in field bioremediation. The results of their studies suggested that nutrient amendment in a high dose can accelerate oil biodegradation and may shorten the treatment period to clean up a contaminated environment.

Biodegradation process is a multi step reaction. Most organisms are capable of only one step transformations and in most cases a complete mineralization of xenobiotic requires the involvement of microbial consortia, in which fungi are supposed to play a very important role. The chlorophenols biodegradation mechanisms conducted by Mucor species are still insufficiently explained. The results presented in this and our previous work (Szewczyk et al. 2003) point to the tested strain as an interesting model for the research on PCP and fuel oil biodegradation and its future application in bioremediation of the areas contaminated with PCP and spent oils. Removal of $55.5 \%$ of initial oil concentration coupled with removal of almost $90 \%$ PCP in only 10 days is a promising result considering the fact that these two processes were conducted by a single strain. The estimation of optimal parameters of PCP biodegradation by Mucor ramosissimus IM 6203 may be a starting point for the application of this method for the bioremediation of areas contaminated with PCP and oils.

\section{Acknowledgments}

This work was supported by University of Lodz grants no. 505/387, 505/488, 505/705

\section{References}

Abramovitch R.A., Capracotta M., 2003. Remediation of waters contaminated with pentachlorophenol. Chemosphere 50, 955-957. Adedokun, O.M.; Ataga, A.E. 2005. Effects of crude oil and oil products on growth of some edible mushrooms. Journal of Applied Sciences and Environmental Management 10, 91-93.

Adenipekun, C.O. 2008. Bioremediation of engine-oil polluted soil by Pleurotus tuber-regium Singer, a Nigerian white-rot fungus. African Journal of Biotechnology, 7:55-58.

Alvarez-Rodriguez M.L., López-Ocańa L., López-Coronado J.M., Rodriguez E., Martinez M.J., Larriba G., Coque J.-J.R., 2002. Cork taint of wines: role of the filamentous fungi isolated from cork in the formation of 2,4,6-trichloroanisole by O-methylation of 2,4,6trichlorophenol. Apllied and Environmental Microbiology 68, 58605861

Arzayus K.M., Dickhut R.M., Canuel E.A., 2001. Fate of atmospherically deposited polycyclic aromatic hydrocarbons (PAHs) in Chesapeake Bay. Environmental Science and Technology 35, 2178-2183.

Atlas R.M, 1981. Microbial degradation of petroleum hydrocarbons: an environmental perspective. Microbiology Reviews 45, 180-209.

Bollag J.-M., 1992. Decontaminating soil with enzymes. An in-situ method using phenolic and anilinic compounds. Environmental Science and Technology 26, 1876-1881.

Bondar V.S., Boersma M.G., van Berkel W.J.H., Finkelstein Z.I., Golovlev E.L., Baskunov B.P., Vervoort J., Golovleva L.A., Rietjens I.M.C.M., 1999. Preferential oxidative dehalogenation upon conversion of 2-halophenols by Rhodococcus opacus 1G. FEMS Microbiology Letters 181, 73-82.

Chaıneau C.H., Morel J.L., Oudot J., 2000. Biodegradation of fuel oil hydrocarbons in the rhizosphere of Maize (Zea mays L.). Journal of Environmental Quality 29, 569-578.

Chaineau C.H., Rougeux G., Yéprémian C., Oudot J., 2005. Effects of nutrient concentration on the biodegradation of crude oil and associated microbial populations in the soil. Soil Biology and Biochemistry 37, 1490-1497.

Copley S.D., 2000. Evolution of a metabolic pathway for degradation of a toxic xenobiotic: the patchwork approach. Trends in Biochemical Sciences 25, 261-265.

Coque J.-J.R., Alvarez-Rodriguez M.L., Larriba G., 2003. Characterization of an inducible chlorophenol O-methyltransferase from Trichoderma longibrachiatum involved in the formation of chloroanisoles and determination of its role in cork taint of wines. Apllied and Environmental Microbiology 9, 5089-5095.

Cortes D., Barrios-Gonzalez J., Tomasini A., 2002. Pentachlorophenol tolerance and removal by Rhizopus nigricans in solid-state culture. Process Biochemistry 37, 881-884.
Garrett R.M., Rothenburger S.J., Prince R.C., 2003. Biodegradation of Fuel Oil Under Laboratory and Arctic Marine Conditions. Spill Science and Technology Bulletin 8, 297-302.

Götz R, Bauer O-H, Friesel P, Herrmann T, Jantzen E, Kutzke M, Lauer R, Paepke O, Roch K, Rohweder U, Schwartz R, Sievers S, Stachel B (2007) Vertical profile of PCDD/Fs, dioxin-like PCBs, other PCBs, PAHs, chlorobenzenes, DDX, HCHs, organotin compounds and chlorinated ethers in dated sediment/soil cores from flood-plains of the river Elbe, Germany. Chemosphere 67:592-603

Hurley M.D., Sokolov O., Wallington T.J., Takekawa H., Karasawa M., Klotz B., Barnes I., Becker K.H., 2001. Organic aerosol formation during the atmospheric degradation of toluene. Environmental Science and Technology 35, 1358-1366.

Janssen D.B., Pries F., van der Ploeg J.R., 1994. Genetics and biochemistry of dehalogenating enzymes. Annual Review of Microbiology 48, 163-191.

Janssen D.B., Pries F., van der Ploeg J.R., 1994. Genetics and biochemistry of dehalogenating enzymes. Annual Review of Microbiology 48, 163-191.

Jiayin D., Muqi X., Jiping C., Xiangping Y., Zhenshan K, 2007. $\mathrm{PCDD} / \mathrm{F}$., $\mathrm{PAH}$ and heavy metals in the sewage sludge from six wastewater treatment plants in Beijing., China. Chemosphere 66 353-361.

Kellner D.G., Mavest S.A., Sligar S.G., 1997. Engineering cytochrome P450s for bioremediation. Current Opinion in Biotechnology 8, 274-276.

Kot-Wasik A., Kartanowicz R., Dąbrowska D., Namieśnik J., 2004. Determination of chlorophenols and phenoxyacid herbicides in the Gulf of Gdańsk., southern Baltic Sea. Bulletin of Environmental Contamination and Toxicology 73, 511-518.

Lamar R.T., Dietrich D.M., 1990. In situ depletion of pentachlorophenol from contaminated soil by Phanerochaete spp. Applied and Environmental Microbiology 56, 3093-3100.

Lamar R.T., Lerson M.J., Kirk T.K., 1990. Sensitivity to and degradation of pentachlorophenol by Phanerochaete spp. Applied and Environmental Microbiology 56, 3519-3526.

Law W.M., Lau W.N., Lo K.L., Wai L.M., Chiu S.W., 2003. Removal of biocide pentachlorophenol in water system by the spent mushroom compost of Pleurotus pulmonarius. Chemosphere 52,1531-1537.

Le Garrec G.M., Artaud I., Capeillere-Blandin C., 2001. Purification and catalytic properties of the chlorophenol 4-monooxygenase from Burkholderia cepacia strain AC1100. Biochimica et Biophysica Acta 1547, 288-301.

Lestan D., Lamar R.T., 1996. Development of fungal inocula for bioaugmentation of contaminated soils. Applied and Environmental Microbiology 62, 2045-2052.

Lobos J.H., Leib T.K., Su T.M., 1992. Biodegradation of bisphenol A and other bisphenols by a gram-negative aerobic bacterium. Applied and Environmental Microbiology 58, 1823-1831.

Machera K., Miliadis G.E., Anagnostopoulos E., Anastassiadou P. 1997. Determination of pentachlorophenol in environmental samples of the S. Euboic Gulf., Greece. Bulletin of environmental contamination and toxicology 59, 909-916.

Michaud L., Lo Giudice A., Saitta M., De Domenico M., Bruni V,, 2004. The biodegradation efficiency on diesel oil by two psychrotrophic Antarctic marine bacteria during a two-month-long experiment. Marine Pollution Bulletin 49, 405-409.

Nascimento N.R., Nicola S.M.C., Rezende M.O.O., Oliveira T.A., Öberg G., 2004. Pollution by hexachlorobenzene and pentachlorophenol in the coastal plain of São Paulo, Brazil. Geoderma 121, 221-232.

Oh Y.-S., Sim D.-S., Kim S.-J., 2001. Effects of Nutrients on Crude Oil Biodegradation in the Upper Intertidal Zone. Marine Pollution Bulletin 42, 1367-1372.

Reddy G.V.B., Gold M.H., 1999. A two-component tetrachlorohydroquinone reductive dehalogenase system from the lignin-degrading basidiomycete Phanerochaete chrysosporium. Biochemical and Biophysical Research Communications 357, 901905.

Sharma V.K., Hicks S.D., Rivera W., Vazquez F.G., 2002. Characterization and degradation of petroleum hydrocarbons following an oil spill into a coastal environment of south Texas, USA. Water Air and Soil Pollution 134, 111-127.

Shim S.-S.., Kawamoto K., 2002. Enzyme production activity of Phanerochaete chrysosporium and degradation of pentachlorophenol in a bioreactor. Water Research 36, 4445-4454.

Szewczyk R., Bernat P., Milczarek K., Dlugonski J., 2003. Application of microscopic fungi isolated from polluted industrial areas for polycyclic aromatic hydrocarbons and pentachlorophenol reduction. Biodegradation 14, 1-8. 
Tsai C.-H., Lin P.-H.., Waidyanatha S., Rappaport S.M., 2001. Characterization of metabolic activation of pentachlorophenol to auinones and semiauinones in rodent liver. Chemico-Biological Interactions 134, 55-71.

Van Hamme J.D., Ward O.P., 2001. Volatile hydrocarbon biodegradation by a mixed-bacterial culture during growth on crude oil. Journal of Microbiology and Biotechnology 26, 356-362.

van Pee K.-H.., Unversucht S., 2003. Biological dehalogenation and halogenation reactions. Chemosphere 52, 299-312.

Venosa A.D., Zhu X., 2003. Biodegradation of Crude Oil Contaminating Marine Shorelines and Freshwater Wetlands. Spill Science and Technology Bulletin Volume 8, 163-178.

Virendra M., Pandey S.D., 2004. Hazardous waste., impact on health and environment for development of better waste management strategies in future in India. Environment International $31,417-431$

Webb M.D., Ewbank G., Perkins J., McCarthy A.J., 2001. Metabolism of pentachlorophenol by Saccharomonospora viridis strains isolated from mushroom compost. Soil Biology and Biochemistry 33, 1903-1914.

Wilmańska D., Milczarek K., Rumijowska A., Bartnicka K., Sedlaczek L., 1992. Elimination of by-products in 11 beta-hydroxylation of substance $S$ using Curvularia lunata clones regenerated from NTGtreated protoplasts. Applied Microbiology and Biotechnology 37, 626-30.

Wright A.L., Weaver R.W., 2004. Fertilization and Bioaugmentation for Oil Biodegradation in Salt Marsh Mesocosms. Water Air and Soil Pollution 156, 229-240. 